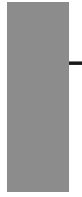

Álvaro Pérez Ragone ${ }^{4}>$

\section{Aportes para Enseñanza del Derecho Probatorio: método colaborativo inducido por el diálogo socrático}

\author{
A Contribution for Teaching Law of Evidence: \\ collaborative method induced by the Socratic dialogue \\ Contribuição para a ensenhanza do Direito Probatório: \\ método colaborativo induzido pelo diálogo socrático
}

Resumen: El método socrático sigue siendo válido -y lo demuestra la investigación más reciente- en tanto complementario a otras metodologías la manera de facilitar al estudiante un tránsito desde el escuchar y olvidar hacia el hacer y saber hacer es el aprendizaje colaborativo con el diálogo socrático. Ello ya que el aprendizaje colaborativo aplicado empíricamente con éxito en la enseñanza con metodologías activas del Derecho Probatoria en el pregrado. Con el empleo combinado del diálogo socrático y las estrategias colaborativas se aprovecha la distracción del estudiante para valorarlo y hacerlo participe en su grupo y en la clase. En el aprendizaje colaborativo la orientación (no imposición) proviene del estudiante y de los pares, el docente no da la respuesta, sino que oriente hacia ella y eso es el plus que otorga lo socrático o inducido al aprendizaje entre pares. El método socrático en el marco colaborativo ha sido redescubierto y demuestra eficacia. Especialmente en la enseñanza del derecho las discusiones en torno a él son muy valiosas y la discusión sobre su aplicación como metodología exclusiva sigue vigente incluso. Este es un aporte para diversificar los métodos de enseñanza del Derecho que no se reducen solo a la ley, su conocimiento y aplicación, la excede y comprende aspectos del estudiante que pueden ser mejor incentivadas y aprovechados. A partir de la determinación del problema en general en la enseñanza-aprendizaje y los desafios planteados, se parte de la hipótesis de la necesidad de combinar el diálogo socrático con el aprendizaje colaborativo como metodología de la enseñanza innovativa y disruptiva. Se traduce en el diseño de actividades y secuencias en un escenario pedagógico real de una asignatura: el De-

$\leftrightarrow$ Profesor de Derecho Procesal Universidad Católica del Norte (Antofagasta/Chile); Universidad San Pablo-T (Tucumán/Argentina).

$\triangle$ alvaro.perez01@ucn.cl-aperezragone@uspt.edu.ar

Revista de la Facultad de Derecho, (45), 2018, e20184506

DOI:10.22187/rfd2018n45a6

ISSN 0797-8316 / elSSN 2301-0665

1 de 29 
recho Probatorio. Los resultados empíricos de esta actividad educativa confirman la validez de las propuestas y tesis planteadas.

Palabras claves: enseñanza, derecho probatorio, colaboración socrática.

Abstract: The Socratic teaching-learning method is still valid - and the most recent research shows it - provided that it is complementary to other methodologies. In this case it is the collaborative learning empirically applied successfully in teaching with active methodologies to teach the Law of Evidence in a undergraduate course. With the combined use of Socratic dialogue and collaborative strategies, the student's distraction is taken advantage of him and make him participate in his group and in the class. In the collaborative learning the orientation (not imposition) comes from the student and the peers, the teacher does not give the answer, but it guides towards it and that is the plus that grants the Socratic or induced to the teachinglearning between pairs. The Socratic method in the collaborative framework has been rediscovered and shows effectiveness. Especially in the teaching of law the discussions around it are very valuable and the discussion about its application as an exclusive methodology is still valid. This is a contribution to diversify the teaching methods of the Law that is not limited only to the law, its knowledge and application, it exceeds it and it includes aspects of the student that can be better incentivized.

Key Words: teaching; evidence law; Socratic collaborative learning.

Resumo: $O$ método socrático ainda é válido - e a pesquisa mais recente mostra isso - desde que seja complementar a outras metodologias. Neste caso, é o aprendizado colaborativo empiricamente aplicado com sucesso em ensinar com metodologias ativas de direito probatório na graduação. Com o uso combinado do diálogo socrático e estratégias colaborativas, a distração do aluno é aproveitada para valorizá-lo e fazêlo participar de seu grupo e na classe. Na aprendizagem colaborativa, a orientação (não a imposição) provém do aluno e dos colegas, a professora não dá a resposta, mas orienta-se para ela e essa é a vantagem que concede aos socráticos ou induzidos a aprender pares. O método socrático na estrutura colaborativa foi redescoberto e mostra eficácia. Especialmente no ensino do direito, as discussões em torno dele são muito valiosas e a discussão sobre sua aplicação como metodologia exclusiva ainda é válida. Este é um contributo para diversificar os métodos de ensino da Lei que não se limita apenas à lei, ao seu conhecimento e à sua aplicação, excede-a e inclui aspectos do aluno que podem ser melhor incentivados e explorados.

Palavras-chave: ensino; lei probatória, colaboração socrática

Recibido: 20180114

Aceptado: 20180302

Revista de la Facultad de Derecho, (45), 2018, e20184506

DOI:10.22187/rfd2018n45a6

ISSN 0797-8316 / elSSN 2301-0665

2 de 29 


\section{Marco teórico, problema, hipótesis, metodología y propuesta}

El alumno no es un recipiente vacío a llenar solo con conocimientos, habilidades, destrezas o concepciones axiológicas. El docente que transmite solo conocimiento puede llegar a ser el "correcto", "usual", incluso "premiado" por sus alumnos. Resulta necesario repensar tres aspectos relevantes en la enseñanza-aprendizaje: 1) Construcción del aprendizaje, 2) con aporte desde las distintas habilidades de cada alumno, 3) para un aprendizaje perdurable. Ello se plantea acá como un problema que admite hipótesis de trabajo y tesis que acá se plantean. Este aporte transita desde lo teórico a la experiencia empírica de la aplicación de la innovación innovativa postulada en el ámbito de la enseñanza del Derecho Probatorio.

Como aclaración previa y necesaria, para el buen desenvolvimiento del proyecto fue necesario que el Área de Derecho Procesal reflexionara detenidamente sobre la metodología que habría de emplear, en aras de maximizar los resultados del proyecto $y$, en definitiva, de que aquel fuera compatible con cada uno de los objetivos formulados. I) El Derecho Probatorio no alcanzó en la tradición latinoamericana (incluso Continental Europea) - salvo excepciones de especialización o cursos optativos de profundización- una autonomía en el grado programática y por ello didáctica y pedagógica (Comp. Nance, 2016, 27). En otra palabras se enseña sobre prueba judicial en el contexto de cursos sobre Derecho Procesal, especialmente al tratar procesos de conocimiento o declarativos. II) Esta falta de autonomía en tiempo y contenidos conlleva a que muchos aspectos se dejen de lado, y que son relevantes para las competencias disciplinares del alumno no solo para el área del procesal (Taruffo, 2003; Leclerc, 2012). III) Normalmente el hilo conductor (con o sin aplicación combinada de clase magistral y algunas metodologías activas como casos, examen de fallos e incluso juicios simulados) por los tiempos de clases y contenidos previos y posteriores al Derecho Probatorio, incentivan acentuar en lo dogmático y jurídico, olvidándose que es relevante para la prueba el acercamiento, reflexión, desgranamiento de detalles fácticos (Nance, 2016; Roberts, 2002) 
El problema abordado en este texto gira en torno a defender, justificar argumentativamente y demostrar con un caso pedagógico la utilidad de un método socrático actualizado y con el uso de las realidades e innovaciones actuales. La hipótesis general a examinar acá sostiene que el diálogo socrático es eficaz y en mayor grado con el complemento de otras metodologías que encauzan al estudiante en el tránsito desde el escuchar y olvidar hacia el saber hacer, ser y estar (Competencia cognitivas, aptitudinales, actitudinales y sociales) (Comp. Hopkins, 2008). Ello se lograría en tanto la metodología propuesta podría coadyuvar a I) incorporar al alumno en la construcción de II) un aprendizaje perdurable III) a partir de sus fortalezas individuales. El aprendizaje colaborativo unido al diálogo socrático podría ser un marco eficiente y eficaz (Lemmer, 2013).

Estas hipótesis generales y especiales son examinadas metodológicamente en forma teórica y empírica por haber sido aplicada la propuesta pedagógicadidáctica en la enseñanza del Derecho Probatorio como asignatura de la carrera de pregrado de Derecho en una Universidad chilena. Así la descripción y diálogos que se exponen entre diversas visiones y doctrina, se evalúan en su aplicación práctica con una curricula específica, y dentro de un escenario educativo concreto, para finalmente ponderar y evaluar las hipótesis, proponen las tesis y resultados de esta investigación aplicada en el ámbito propio de la didáctica jurídica (nuevas metodología activas para la enseñanza-aprendizaje del Derecho). A partir del diseño del programa de la asignatura desde la taxonomía de Bloom (planificación) (así se trabajó el diseño del programa de la asignatura a la que se aplica este proyecto que considera las revisiones a los aportes originales de Bloom a partir de (Anderson, Krathwohl et al., 2001; Heer, 2012)), fue posible implementar en cada clase el ciclo descrito por Kolb (implementación) (Kolb, 1976; Kolb y Kolb, 2005). Lo primero (postulado taxonómico) determina el diseño en resultados, contenidos y actividades semestral, lo segundo (Ciclo de Kolb) su concretización en cada momento de interacción docente-alumno (Wolff y Chan, 2016, 35; Schatzberg, 1999). En la implementación se fijan marcos flexibles para generar espacios de interacción entre los estudiantes y de ellos con el docente. Se incentiva el aprendizaje colaborativo y diálogo socrático que permite potenciar habilidades cognitivas y metacognitivas (Bartimote-Aufflick, y Ainle, 2006; Mayer, 2009; Mazur, 1996). 
Mediante estas líneas se pretende demostrar que la aplicación del método socrático en la enseñanza de una disciplina jurídica especial como el Derecho Probatorio sigue siendo útil y válida. Ello unido a otras metodologías complementarias como la colaborativa, el planteo de problemas y el interrogatorio orientador y empático para un adecuado ambiente o escenario del aprendizaje. Justamente luego de tratar los marcos teóricos argumentativos y la descripción de los principales núcleos de la asignatura, se describe una experiencia concreta de aplicación de aquellos que pretende ser un aporte en la diversificación para un proceso de enseñanza- aprendizaje efectivo y eficiente (Alford 2008). Una perspectiva histórica sobre el papel del método socrático tiene defensores y detractores en la en la actual batalla por la reforma curricular. En realidad en la aplicación de esta metodología tiene casi mil años en la tradición occidental sajona, germana y francesa. Tiene sus raíces en el Derecho Romano, arraigamiento en la Escolástica y perduró por siglos con éxito Universidad de París de esa época, y es un error platear que su creación se debe a la brillante iniciativa de cambio curricular y propuesta metodológica de Langdell en Harvard (Alford 2008). En este texto no se profundizan conceptos en general como la "pedagogía", didáctica" o "metodología", se parte de su existencia y comprensión para hacer aplicación en concreto mediante la innovación que se propone en un área especial del Derecho. Este es un texto esencialmente interdisciplinario y por ende acude a fuentes de ciencias de la educación, jurídicas en general, de didáctica jurídica en particular, de Derecho Comparado y específica del área del Derecho Probatorio ${ }^{1}$.

Este aporte se compone de una primera sección en el que se describe el complejo mundo del la prueba judicial y su regulación mediante el Derecho Probatorio en forma general para la comprensión por el lector de aquello que será objeto de auscultamiento didáctico y pedagógico (II); se continúa con el marco donde se propone la aplicación de innovaciones pedagógicas y didácticas del Derecho (III); luego se describe, explora y analiza el desarrollo de las actividades que importan aplicar la propuesta acá planteada (IV); se cierra con conclusiones en base a lo desarrollado y la experiencia práctica de aplicación realizada y a su vez se insta de futuro a aplicar metodologías innovativas activas evidenciadas como eficaces por la literatura y el caso didáctico propuesto en este artículo para su uso (V). 


\section{El fascinante y complejo mundo de la Prueba Judicial}

El proceso judicial busca adjudicar en justicia en base a información que se considera acreditada o no. Dado que el proceso es complejo en quienes lo integran y las posiciones que en él se defienden, el juez no puede pronunciarse tan sólo con lo que las partes alegan o afirman, ya que en muchos casos y de acuerdo a sus intereses lo que afirme una será controvertido por la otra ${ }^{2}$. Se trata pues de verificarlas afirmaciones de las partes, las que deben ser corroboradas, constatadas o evidenciadas. Se sostiene que la prueba tiene como objetivo formar la convicción del juzgador fundado en un nivel o peso en la credibilidad de la evidencia empleada para ello. El proceso judicial trabaja con la incertidumbre e incerteza de la veracidad de las alegaciones fácticas de las partes. La prueba judicial debe lidiar con la incertidumbre, el tiempo acotado en el cual debe brindarse una respuesta jurisdiccional y el hecho que esa respuesta será vinculante y no revisable como podría ser un resultado en un laboratorio. Solo excepcionalmente una decisión judicial firme es revisable y rescindible (Nance, 2016).

¿Para qué sirve la prueba? Existen al menos dos alternativas teóricas examinables respecto a la finalidad de la prueba (Taruffo, 2010):

a) Búsqueda de la verdad consensual: Esta posición se relaciona con la teoría de la verdad de corte habermasiano o la corriente más contemporánea de la epistemología social, de manera tal que el nivel de racionalidad comunicativa de la prueba está dado por el consenso que puede producir en un grupo o comunidad. La crítica que se le puede efectuar a esta posición es que no es aplicable a la vida real, en la medida en que es impracticable en una sociedad que no exista un discurso libre de dominación y un presupuesto de igualdad entre las partes. O bien la verdad se busca y construye con el actuar comunicativo de las partes y el juez en el proceso, excede las condiciones epistémicas de un simple sujeto que conoce un simple objeto.

b) La verdad no puede ser buscada y el proceso por sí garantiza un resultado que siempre será correcto. La legitimidad del proceso es suficiente para la legitimidad del resultado, neutro a cuestiones epistémicas y valóricas. Se basa, principalmente, en que históricamente los procesos no han buscado la verdad -como en el caso de las ordalías o juicios de 
Dios-, sino que intentan resolver un conflicto para devolver la paz social. Parte de esta teoría es sostener que la legitimidad de la decisión la provee el propio proceso, y que ello es independiente de la verdad, ya que la legitimidad misma de la decisión la otorga el haber respetado las reglas procedimentales para llegar a una respuesta jurisdiccional. Ésta es neutra y la verdad le resulta irrelevante, el respeto del proceso garantizaría pues la legitimidad del resultado e independiente de la realidad.

La prueba tiene dimensiones epistémicas y normativas que la convierten en un área fecunda y compleja al mismo tiempo. Moverse en esas dos dimensiones puede llevar a un mejor o peor sistema probatorio. Claro que sería fácil partir con un escepticismo absoluto y solucionar el problema ya que se podría sostener que no se puede llegar a ningún tipo de verdad. También podría optarse por términos intermedios sosteniendo que es posible el acercamiento a una realidad que podrá o no ser replicada. Se podrá así hablar de estándares de acercamiento a la verdad que permiten exigir cierta objetividad en la explicación del convencimiento judicial en la decisión como parte de su debida motivación (Fredrick, 2010). Desde una ignorancia absoluta, pasando por exámenes de verosimilitud, hasta llegar a un nivel de probabilidad que quizás sea suficiente para la certeza del juez, se van incrementando los niveles de conocimiento. Nos encontraremos así con varios escenarios posibles: (I) Un absoluto "convencimiento" de lo ocurrido sustentado además en evidencia, de forma que es posible decir que tales o cuales hechos acontecieron tal cual lo describe tal o cual parte; (II) Un absoluto estado de "duda" sobre cómo acontecieron los hechos que me impide llegar a una conclusión; (III) Un relativo "convencimiento" mayor o menor, en base al mérito demostrado por las partes (Fredrick, 2010). En materia civil, simplemente se establece un criterio de «más allá de toda probabilidad no acreditable». Un estándar aceptable en materia civil es 0,5 o de $50 \%$ siendo más exigente en materia penal (más allá de toda duda razonable) que reflejan en realidad un nivel mínimo de probabilidad preponderante.

Es posible sostener que la prueba tiene por finalidad establecer la veracidad de las afirmaciones que los intervinientes realizan en un juicio. Ello se condice a su vez con la teoría de la verdad como correspondencia, que busca que las afirmaciones vertidas por las partes se correspondan con la realidad, con los hechos, siendo la prueba la forma de acreditar ello. Esta posición se corresponde con la que considera que el convencimiento del juzgador no se 
asienta en la visión íntima o subjetiva, sino que puede expresarse en tanto correspondencia entre lo afirmado y lo corroborado (probado). Para ello los sistemas establecen una serie de regulaciones o sistemas de reglas sobre la prueba que versan acerca de: a) las fuentes o elementos de prueba admisibles en el juicio; b) los medios de prueba, que son instrumentos que permiten dar por acreditadas las afirmaciones como verdaderas y que se usan para traer al juicio las fuentes de prueba; y c) los sistemas de valoración de la prueba, que son modelos indicados por el legislador en los cuales el juez debe desarrollar su actividad para dar por acreditada esas afirmaciones, y de las limitaciones probatorias (Sentis Melendo, 1979).

En el contexto de la regulación de la prueba, es dable distinguir normas epistemológicas y normas contra-epistemológicas. Las primeras son cualquier elemento que sirva para establecer la veracidad de las afirmaciones emitidas por las partes. Las segundas permiten conocer la veracidad o falsedad de las afirmaciones, pero traspasan los principios y valores superiores para su obtención, por lo que no se pueden incorporar al proceso, tal es el caso de la prueba ilícita presentada en juicio o los casos donde el propio legislador ha determinado que un medio de prueba en determinadas circunstancias no es admisible (por ejemplo el principio de prohibición de autoincriminación o la prohibición de declarar de ciertos testigos sobre información confidencial en razón de su oficio) (Nance, 2016)

Carnelutti conceptualiza a la prueba como "comprobación de la verdad de una proposición, sólo se habla de prueba a propósito de alguna cosa que ha sido afirmada" (Carnelutti, 1982). En la misma línea Couture sostiene la prueba civil es “...comprobación, demostración, corroboración de la verdad o falsedad de las proposiciones formuladas en el juicio" (Couture, 1977) Los hechos que se consideran en el proceso son aquellos que cada parte introduce como los que considera relevantes y decisivos para fundar su caso. El juez en ello no interviene ya que son las partes las que determinan su caso. Un análisis más detallado en realidad debe partir por examinar lo que sostiene el demandante en primer lugar (estadio del demandante), para luego hacer lo propio del demandado (estadio del demandado). Cada parte incluirá en su discurso hechos relevantes o no, incluso algunos revestidos como si fueran materia de derecho y no de hecho. Un tercer análisis será el probatorio. Acá es donde debe decidir el juez sobre las diferentes alegaciones relativas a los hechos que efectuaron las partes, cuáles de todos ellos resultan ser relevan- 
tes y a su vez contradictorios para ser justamente referidos y sometidos a la actividad probatoria (Prütting, 2016).

\section{Marco de aplicación de innovación en la enseñanza del derecho probatorio}

Derecho Probatorio ${ }^{3}$ es una asignatura que se imparte dos días a la semana, cada sesión de 180 minutos, incluyendo un recreo de 10 minutos y examina los principales contenidos descritos en la sección anterior. Las actividades de enseñanza y evaluación propuestas en este proyecto comprenden un total de cinco sesiones (dos semanas y media en total de duración presencial y no presencial). La evidencia judicial sirve para justificar y convencer sobre un relato o visión tanto a un juez como a la contraparte. Los contenidos a explorar comprenden: (I) concepto, función y objeto de la prueba, (II) la actividad probatoria, (III) tipos de prueba, (IV) examen y valoración de la prueba por el juez al momento de decidir responsabilidad de los abogados por la justicia de sus causas. Esta decisión debe estar motivada o fundada, explicitar por qué considera creíble el relato de una u otra parte. Una sentencia motivada legitima al juzgador ya que hay una íntima relación entre prueba, verdad y justicia.

Cada sesión presencial se inicia con una pregunta sobre cómo se sienten en ese día (construcción de clima de confianza y distención); continúa con una breve introducción que resume lo realizado en la sesión anterior relacionándolo con los contenidos y actividades de la actual; sigue el desarrollo de la clase (proyección de video con diálogo socrático y/o trabajos en grupo según la sesión); se concluye la clase con un resumen de lo realizado y anuncio de lo que se hará en la clase siguiente. La secuencia de aprendizaje compren de sesiones con proyección de una película, trabajos en grupo, reformulación y examen grupal de la respuestas, lecturas para concluir en una actividad co-evaluativa con su retroalimentación (Posner, 2001; Fiss, 1999) (Ver anexo 1 al final).

Debe tenerse en cuenta que las explicaciones y desarrollo de un programa en una asignatura y la sustanciación de un proceso judicial tienen un elemento común: su desarrollo en un tiempo limitado. La clave del éxito de esta experiencia radica en "sincronizar ambos relojes" para que mantengan un ritmo armónico: el tiempo de los contenidos en enseñanza-aprendizaje efec- 
tivos y el cronológico. El manejo de los tiempos de las sesiones presenciales se realiza siempre de acuerdo al ciclo de Kolb (percepción-reflexión-conceptualización-aplicación). La metodología empleada para la enseñanzaaprendizaje combina el método socrático, aprendizaje colaborativo, método del caso y el ABP (Aprendizaje Basado en Problemas), se entiende por "problemas" las situaciones fácticas que se van presentando relacionadas con los fundamentos de la prueba judicial) (Ryan, Shuai, Ye, y Ran, 2014)

Resulta pertinente acá este cuadro comparativo entre el "caso" y el "problema" para aclarar el rol que asumen cada uno de los agentes. Por la dinámica que se imparte, se puede decir que el caso en nuestro experimento didáctico fueron las situaciones simples a resolver planteadas con un contenido específico del programa (Berret 2012). Mientras que el problema consistió en una situación compleja que impone relacionar varias situaciones ya analizadas como "casos" para vincular temáticamente varios contenidos interrelacionados del programa de la asignatura. (ver tabla 1)

Por ejemplo, ellos ofrecen un excelente análisis de cómo la lógica Bayesiana podría ayudar a los sujetos a cargo de determinar los hechos (factfinders) a resolver controversias reales. Además, enseñar a los estudiantes a examinar la regulación probatoria acudiendo a elementos interdisciplinarios para concentrarlos en la perspectiva procesal. La concentración en los alegatos de cierre del juicio de la película, por ejemplo, es un momento sucinto, preciso y lleno de detalles del Derecho Probatorio para analizar. El detalle de un proceso judicial en formato totalmente diferente del nacional ayuda a incorporar dudas e instituto del Derecho Procesal Comparado: un sistema de audiencias, cuando en Chile es escrito totalmente, un sistema donde hay un jurado, un sistema donde la publicidad del caso genera externalidades en la sociedad donde se lleva adelante versus el secretismo del procedimiento escrito etc. (Rothstein, 2006). La narración de una historia (en el caso acá propuesta una película que impacta en la percepción multisensorial y emocional) es un antídoto para que los estudiantes se alejen indirectamente de la mera memorización y simple regurgitar de reglas mecánicamente sin entender el propósito o el contexto de la ley. La narración no valida el mito del "análisis objetivo", la suposición de que la doctrina es la unidad básica de análisis de la realidad. Por el contrario, la narración de historias demuestra que la experiencia humana es la fuente para el desarrollo de la ley. Cuando se articulan los motivos de la política o los propósitos subyacentes de las reglas, se dan 
estructura y significado a las reglas. Por lo tanto, contar historias contextualiza las reglas de maneras que muestran que, en realidad, los hechos están llenos de valores y no de valor neutral (Beryl, 1992)

\section{Tabla 1}

\begin{tabular}{|l|l|l|}
\hline \multicolumn{1}{|c|}{ Aspecto de análisis } & \multicolumn{1}{|c|}{ Método del caso } & \multicolumn{1}{c|}{$\begin{array}{c}\text { Apendizaje basado } \\
\text { en problemas }\end{array}$} \\
\hline $\begin{array}{l}\text { Situación planteada } \\
\text { y percibida }\end{array}$ & Real & Real o ficticia \\
\hline Reflexión sobre la situación & $\begin{array}{l}\text { Secuencia: } \\
\text { Individual / pequeño grupo / } \\
\text { gran grupo / pequeño grupo }\end{array}$ & $\begin{array}{l}\text { En grupo desde el principio } \\
\text { con una fase de trabajo indi- } \\
\text { vidual }\end{array}$ \\
\hline $\begin{array}{l}\text { Características del caso } \\
\text { y del problema }\end{array}$ & $\begin{array}{l}\text { No existe una única solución } \\
\text { correcta }\end{array}$ & $\begin{array}{l}\text { No existe una única solución } \\
\text { correcta }\end{array}$ \\
\hline $\begin{array}{l}\text { Información con la que } \\
\text { cuentan los alumnos }\end{array}$ & $\begin{array}{l}\text { Se presenta, en la mayoría de } \\
\text { los casos, toda la informa- } \\
\text { ción necesaria para las solu- } \\
\text { ciones posibles }\end{array}$ & $\begin{array}{l}\text { Generalmente los alumnos } \\
\text { tiene que ampliar la informa- } \\
\text { ción y relacionar la existente }\end{array}$ \\
\hline $\begin{array}{l}\text { Papel profesor en el diálogo } \\
\text { socrático }\end{array}$ & $\begin{array}{l}\text { Guía del conocimiento pre- } \\
\text { vio y del conocimiento gene- } \\
\text { rado a través del debate y la } \\
\text { discusión a partir del diálogo } \\
\text { socrático }\end{array}$ & $\begin{array}{l}\text { Orientador de búsqueda de la } \\
\text { información y orientador en } \\
\text { el proceso de solución. Inter- } \\
\text { acción con el alumno siem- } \\
\text { pre a partir del diálogo } \\
\text { socrático }\end{array}$ \\
\hline $\begin{array}{l}\text { Alumnos en el aprendizaje } \\
\text { colaborativo }\end{array}$ & $\begin{array}{l}\text { Se trabaja en grupos en cla- } \\
\text { ses y se hace dialogar a los } \\
\text { miembros del grupo entre sí } \\
\text { y a todos los grupos }\end{array}$ & $\begin{array}{l}\text { Se trabaja en grupos fuera de } \\
\text { clase orientándolos en base a } \\
\text { la experiencia que tuvieron } \\
\text { en las clases. Se orienta pre- } \\
\text { sencial o virtualmente a los } \\
\text { grupos por separado. Sirve } \\
\text { para sintetizar lo elaborado } \\
\text { en clases y preparar la si- } \\
\text { guiente clase }\end{array}$ \\
\hline
\end{tabular}

No obstante las diferencias de contexto, lo que los une: mostrar a los estudiantes algunos de los problemas que los abogados litigantes y jueces enfrentan diariamente es, en mi opinión, indispensable para ensenar derecho probatorio exitosamente. La incertidumbre del juez que debe ser convencido de un relato de acuerdo a determinadas reglas. A partir de estas metodologías 
aplicadas oportuna y adecuadamente puede formarse al alumno en los fundamentos del Derecho Probatorio para luego avanzar casos y problemas complejos de la disciplina. Aún el estudiante no puede plantear estrategias probatorias, menos dirigir un interrogatorio o repreguntar a testigos, peritos o a las partes. Tampoco ha examinado y valorado el conjunto de la prueba rendida, ni tampoco experimentó la incertidumbre de lo realmente acontecido. En realidad sabe por la película misma qué aconteció y cómo, la duda e incertidumbre que rememora el alumno tiene pequeñas lagunas y es consciente de lo que trata cada parte de ser presentada al juez y jurado, según la parte y sus intereses. Todo ello lo ha visto en la película, lo ha "percibido" e incluso reflexionado el alumno, pero no son resultados del aprendizaje esperados en estas unidades generales e introductorias del programa (Ryan, Shuai, Ye y Ran, 2014; Kolb y Kolb, 2005)

La innovación metodológica en la enseñanza en este caso del Derecho Probatorio puede medirse por la cantidad de materiales empleados en sustitución de manuales dogmáticos sobre la prueba judicial y en indicadores de seguimiento y rendimiento del alumno. Hay lecturas orientadas de textos específicos y generales seleccionados por el docente pero que acompañan la fase de percepción y reflexión para tener más relevancia en la conceptualización. Esto se realiza inicialmente de modo que el alumno puede ir descubriendo individualmente y en grupo tópicos a los que aplica y nuevamente se genera el ciclo con nuevos ingredientes bajo la orientación del docente. Para una fase posterior a esta de introducción, el alumno también se topará con decisiones de los tribunales sobre aspectos de la prueba que permitirán profundizar y consolidar lo visto. Casos y problemas más complejo que se planteen instarán la memoria por analogía de modo que el docente pueda orientar al alumno en la propuesta de soluciones más elaboradas (Lam, 2004).Un problema común es el establecimiento de resultados poco claros o demasiado amplios para cubrirlos de manera realista. Es una buena práctica enfocar el aprendizaje al expresar los resultados como preguntas. Esto proporciona dirección a los estudiantes y ayuda a alcanzar un nivel más profundo de comprensión durante el estudio privado. La otra ventaja de redactar los resultados como preguntas es tener la certeza que la tarea ha sido realizada cuando ha encontrado una buena respuesta. No hay una respuesta absoluta sino una pluralidad de buenas respuestas finalmente sistematizarse en la retroalimentación constante. Esta es una buena forma de evitar la sobrecarga de trabajo. 
El elemento gatillador de la atención del alumno en todas las clases y reuniones de tutorías se logran con el uso del método socrático. Los nuevos métodos de enseñanza empleados son un esfuerzo iniciado para dar a los estudiantes la oportunidad de aprender a través de situaciones en las que se los incluye en situaciones simuladas (no se refiere con ello a los tradicionales "juicios simulados" que son un útil ABP), sino en circunstancias parecidas a los que se presentan a los abogados en diversas áreas. Esta formación en la introducción y posterior profundización en el Derecho Probatorio sirve para asignaturas posteriores en la malla curricular como los distintos tipos de clínica que trabajan con casos y problemas reales para los cuales el alumno ya viene preparado de un ambiente simulado como el planteado en este curso (Kaori, Hidetoshi, 2017).

El docente asume varios roles: interviene sistematizando el aporte, planteando dudas y orientando hacia las respuestas (Thies, 2010). Como facilitador conduce con preguntas el trabajo individual dentro del grupo, de los grupos entre sí y de cada alumno. El método de diálogo socrático permite estimular, conducir, retroalimentar y concentrar la discusión. En los modelos de los modelos de preguntas socráticas que orientan al alumno grupal o individualmente hacia la respuesta buscada se incorporan perfectamente los verbos claves propuestos por Bloom , en el caso de esta asignatura solo para el proceso cognitivo en los tres dominios iniciales del conocimiento, comprensión y aplicación (Wolff y Chan, 2016; Ryan, Shuai, Ye y Ran, 2014). Además valora el aporte individual y grupal, motiva el desempeño desde las particularidades de cada alumno para la obtención de un resultado común y así la maximización del aprendizaje. Finalmente el docente aprecia y pondera los resultados obtenidos, retroalimenta y adecua sus estrategias (Miller, Schell, Ho, Lukoff, y Mazur, 2015; Fadul, 2009). El diálogo con los alumnos se realiza mediante el método socrático que crea un foro seguro que cobija el error y el acierto para ofrecer beneficios sustanciales a partir del aprendizaje desde una visión constructivista de la educación (Ryan, Shuai, Ye y Ran, 2014) (Ayers, 2006).

\section{Desarrollo}

La enseñanza del derecho está fuertemente asociada con el aprendizaje casi memorístico para satisfacer mínimos de evaluación. Así no genera ni motivación, ni aprendizaje perdurable (Thalluri, O' Flaherty, y Shepherd, 2014). Esta asignatura es interdisciplinaria: abarca aspectos epistémicos 
(Gnoseología), de ejercicio de la retórica ponderación de la credibilidad del relato (Psicología Jurídica), y también del poder y su legitimidad (Filosofía Política y Social). Con ello es un área propicia para estas propuestas de innovación metodológicas (Kennedy, 1982). Convencer sobre la verdad de un relato involucra ponerse en el lugar del interlocutor de ese diálogo (empatía), mucho más complejo en un contexto epistémico en el que se plantean dos o más narraciones y justificación de las mismas a ser examinadas por un tercero como es el juez. Insta al docente a esforzarse por involucrar al alumno en la comprensión y contextualización del tema remarcando la importancia que tiene en su desempeño profesional futuro el valor de justicia de la causa que representan (Kennedy, 1987).

El aprendizaje colaborativo es aquella estrategia y metodología de enseñanza-aprendizaje en la que los alumnos asumen un rol compartido para un fin común (Palincsar, 1998). El docente planifica el qué y para qué de la actividad de aprendizaje (de allí lo de "estrategia"), pero además sabe de antemano el uso que hará de ella para combinar paulatinamente según los resultados esperados de la asignatura solo determinados procesos cognitivos, procedimentales y metacognitivos (Pantoja Ospina, 2013; Gokhale, 1995). La tarea de preparación para cada clase es asumida por el alumno y acompañada por el docente que debe: prever la interacción entre pares dentro y fuera de la sala de clases; que algunos alumnos asistirán a la clase con las lecturas indicadas y otros no; orientar y proveer información para concentrar al alumno en lo que se espera de él (Coloma Correa, 2005; Hess, 2013). Esta propuesta tiene como objetivo implementar, examinar, validar, corregir y dar a conocer las potencialidades del uso combinado de metodologías activas de aprendizaje. Al aprendizaje colaborativo se suma otra herramienta: el diálogo socrático. Con ello se logra que el alumno pueda "aprehender" a partir de la percepción y reflexión, conceptualización y aplicación paulatina de habilidades cognitivas y actitudinales (Miller et al., 2015).

La investigación ha puesto de manifiesto la importancia de involucrar varios órganos de los sentidos para una mejor experiencia de aprendizaje dentro del marco cooperativo de aprendizaje (Mazur, 2006). El docente siempre está disponible para asistir a los alumnos individual y grupalmente, recopila información de seguimiento para adecuar las estrategias de enseñanza-aprendizaje (Strayer, 2012, 172; Lage, Platt, y Treglia, 2000). El cuidado en la implementación desarrollada en los últimos 30 años de diseños con la visión 
propuesta de integración de metodologías activas se denomina "Flipped Learning", "Blended Learning" o simplemente clase invertida. En la literatura más contemporánea se hace resalta el uso de internet y metodologías on-line que se excluye de este proyecto (Wolff, y Chan, 2016).

El aprendizaje colaborativo genera el escenario de confianza en el cual los alumnos puedan hacer y saber hacer con sus pares orientado con diálogo socrático que inculca el docente (Vickrey, Rosploch, Rahmanian, Pilarz y Stains, 2015). El docente diseña el escenario y proporciona los recursos necesarios coherentes para el resultado del aprendizaje de un contenido. El uso de la metodología de aprendizaje cooperativo complementado con la clase socrática coadyuva a obtener excelentes resultados (Prince, 2004; Gokhale, 1995). La clase socrática necesita insumos previos como saber de cada alumno cómo desea aportar a la clase, ello para permitir que todos participen, incluso aquellos que lo hacen más pasivamente. En el caso de esta asignatura el docente solicitó a los alumnos respondan unas preguntas indicando cómo deseaban participar en las clases, en ello se siguió la recomendación de cómo conocer mejor a los alumnos (Hess, Friedland, Schwartz, y Sparrow, 2011). Se solicita a los alumnos señalen como desean participar en clases (con nombre y por escrito): a) Siendo interrogados sin previo aviso ; b) Interrogados con previo aviso; c) Disertando con tareas asignadas con antelación; d) Respondiendo como parte de un grupo; e) Respondiendo presentando una minuta o resumen de la clase por escrito. Ello se realizó en esta clase de modo que el docente puede saber las preferencias de cada alumno.

Las preguntas a formular en los distintos escenarios didácticos están previamente diseñadas por el docente, es un proceso muy disciplinado que actúa como el equivalente de la intuición interna del alumno al que orienta en las habilidades de pensamiento crítico. Las preguntas socráticas combinan aspectos del proceso cognitivo (recuerdo, comprensión y aplicación) como metacognitivo (fáctico, conceptual, procedimental y metacognitivo) siguiendo las recomendaciones en esta materia (Heer, 2012). Así por ejemplo la pregunta al grupo: ¿Reconocen sobre qué discuten las partes en la película? Se busca una respuesta que combina el aspecto conceptual a partir del recuerdo de la percepción (lo visto y oído). Las contribuciones de cada alumno individual o como parte de un grupo son valoradas en ese camino ya que el acierto y el error sirven para la orientación, cada aporte debe ser tratado con equidad y cuidado (Ryan, Shuai, Ye y Ran, 2014). La labor del profesor sigue siendo 
muy desafiante en este contexto, pues es él quien con mucha delicadeza deberá ir orientando la discusión y, si es necesario, proveyendo de nueva información que no está disponible para los estudiantes. Así se evita el que todo discurso termine siendo relativizado (Turull, 2011) (Cárdenas, 2002).

Esta actividad de aprendizaje y evaluación comprende cinco sesiones, cada una en dos partes con recreo de por medio. La actividad no presencial del alumno es de dos horas totales (lectura de guía y respuestas a preguntas en forma individual) El alumno ya en la primera clase se le comunica el material de lectura para la asignatura. En las sesiones se va recordando el material de lectura correspondiente

La secuencia de las actividades de aprendizaje comprende (Ver anexo 2):

a. Proyección de película (primera parte). En la primera sesión se inicia con el elemento motivador de compartir la primera parte de una película con los alumnos. La película (Filadelfia (1993)) fue seleccionada previendo sea desconocida para los alumnos por su rango etario, tenga por protagonistas a abogados y desarrolle además temas relacionados con proceso judicial. Previo recreo se conforman cuatro grupos de trabajo con preguntas sobre la película que deben ser incluidas en una matriz para simplificar la actividad. Se insta así el trabajo en grupo en torno a preguntas con matrices sobre lo tratado para centrarse en la percepción o experiencia (focalización en la explicación sin usar lenguaje técnico). La clase se cierra resumiendo el docente los aportes de cada grupo incorporando paulatinamente leguaje técnico y se invita a la lectura de un texto y respuesta a un cuestionario individual para la clase siguiente (Recursos didácticos película, actividades en grupo para completar una matriz; Rol docente de acompañamiento y motivacional de las actividades en grupo valorando los aportes individuales)

b. Revisión en pareja de control de lectura con proyección de preguntas y competencia entre las parejas (actividad evaluativa sin nota): preguntas con respuestas abiertas y selección múltiple. El docente presenta diapositivas con las preguntas del cuestionario reformuladas como alternativas múltiples y va solicitando a cada pareja su opción espera el diálogo y corrección mutua entre los miembros sobre la respuesta correcta (Mazur, 2006) En la dispositiva el docente va resaltando las op- 
ciones que se proponen, al final señala cuál es la correcta. Se procura a partir del acierto y error puedan perfeccionar sus respuestas cada pareja (Recursos didácticos lectura, actividades en parejas y cooperativo, diapositivas con el cuestionario entregado; Rol docente de propone las preguntas según cuestionario diapositivas, recopila las respuestas, insta al diálogo entre los miembros de las parejas, propone y fundamenta la respuesta correcta).

c. Se suma una sesión de sistematización y mapas conceptuales de los contenidos trabajados hasta ahora (focalización sobre reflexiones y conceptos técnicos). La clase se desarrolla en torno a construir y consolidar el proceso de conceptualización y hacer partícipe a cada grupo y pareja participante de las etapas previas. El docente conduce al curso activamente desde la percepción, por la reflexión hasta la conceptualización (Recursos didácticos lectura, diapositivas con mapas conceptuales uso de Prezi; Rol docente expositivo, pregunta y orienta en las respuesta en clase, sistematiza).

d. Película (segunda parte) y trabajo en grupo en torno a preguntas con matrices sobre lo visto (focalización sobre conceptualizaciones y aplicación de ellas). Un proceso judicial donde pueden comenzar a aplicar lo que se conceptualizó. Se desarrolla luego una actividad grupal. Vuelven a conformarse los cuatro grupos con asignación de tareas en torno contenidos específicos del programa en los que deben usar ahora lenguaje técnico. El docente acompaña a cada grupo y los asiste en sus actividades. Luego hace una sistematización con mapas conceptuales sobre cada tema. Se cierra la clase invitando a los alumnos a participar en la siguiente sesión que es evaluativa. Se aprovecha para explicar al alumno como será su rol como evaluado, co-evaluador y examen de desempeño en ambas actividades que realizará el docente mediante una rúbrica. Se entrega la rúbrica y un modelo del quiz (Recursos didácticos película, actividades en grupo para completar una matriz; Rol docente de acompañamiento y motivacional de las actividades en grupo valorando los aportes individuales). 
La secuencia de las actividades de evaluación se compone de los siguientes pasos (Ver anexo 3):

a. El control tiene cuatro temas cada uno con 4 preguntas. En 30 minutos deben responder las preguntas. Luego el docente redistribuye los controles a los alumnos con un tema diferente para que lo corrijan en otros 30 minutos. Así cada alumno finalmente tratará un total de 8 preguntas en base a lo original asignado más lo que debe coevaluar. De esta forma se combina una medición formativa con otra coevaluativa (Hammill, Best y Anderson, 2015).

b. En la siguiente sesión el docente devuelve los controles escritos con las notas de desempeño original, como coevaluador, a quienes se invita ubicarse en parejas.

c. Se analiza en una hora proyectándose cada pregunta e invita el docente al curso a que propongan las respuestas correctas, se las explica y también se identifican las opciones o propuestas incorrectas. De esta forma en la retroalimentación el alumno tiene un rol activo y entre las parejas también se enseñan recíprocamente.

d. El docente valora el rendimiento individual, en parejas y colectivo. Se cierra con un mapa conceptual de repaso de los contenidos.

La actividad evaluativa pretende obtener indicadores del proceso de experiencia, reflexión, conceptualización y aplicación mediante la combinación del aprendizaje colaborativo con la clase socrática (Lobato, 1997; Nicol y Boyle, 2003). Mediante la coevaluación el alumno puede examinar las respuestas de su compañero, aprender de ella, proponer correcciones. De esta forma hay cooperación durante el aprendizaje y en la actividad evaluativa (Thalluri, O' Flaherty y Shepherd, 2014) (Cobas Cobiella, 2015). 


\section{Conclusión en perspectiva y prospectiva}

La excelencia del aprendizaje es resultado de la excelencia de la enseñanza. Para generar un aprendizaje perdurable se consideran los dominios cognitivos y del conocimiento que se entrecruzan y son dinámicos (Bloom), para implementar ello en cada actividad se considera el ciclo de Kolb incorporando las metodologías activas del aprendizaje colaborativo orientado con el diálogo socrático en cada sesión. En relación a objeciones eventuales a esta propuesta sobre el "diálogo socrático":

a. El método socrático sigue siendo válido -y lo demuestra la investigación más reciente- siempre que sea complementario a otras metodologías. En este caso lo es al aprendizaje colaborativo. Quizás la observación realizada pueda ser válida en el contexto de la aplicación exclusiva del "interrogatorio socrático" sin combinar con otras metodologías. Así el diálogo socrático usa y aprovecha la distracción del estudiante para valorarlo y hacerlo participe en su grupo y en la clase. En el aprendizaje colaborativo la orientación (no imposición) proviene del estudiante y de los pares, el docente no da la respuesta, sino que oriente hacia ella y eso es el plus que otorga lo socrático o inducido al aprendizaje entre pares (Ryan, Shuai, Ye y Ran, 2014) (Ayers, 2006; Santiveri, Iglesias, Gil, y Jordana, 2011).

b. El método socrático en el marco colaborativo ha sido redescubierto y demuestra eficacia (Oh y Reamy, 2014) (Strayer, 2012). Especialmente en la enseñanza del derecho las discusiones en torno a él son muy valiosas y la discusión sobre su aplicación como metodología exclusiva sigue vigente incluso (Cobas Cobiella y Mirrow, 2014).

c. Efectivamente como método único ha demostrado su fracaso, ahora "compasivo" y en ambiente colaborativo por el contrario evidencia éxito (Wolff y Chan, 2016, 43-55). Como método único y en la versión tradicional inquisitiva puede es una opción no eficaz considerando la nueva generación de estudiantes que ingresan a las salas de clases. Distinto es su aplicación complementaria al aprendizaje colaborativo para orientar y encausar individual y grupalmente al/a los alumnos. La combinación con el aprendizaje basado en proyectos y el método del caso dan cohesión y encausan amigablemente la enseñanza en la propuesta 
pedagógica y didáctica acá formulada (Orejundo, Fernández y Garrido, 2008) (Fernández March y Bolonia, 2006).

El propósito declarado en el programa de la asignatura mediante resultados de aprendizaje podría ser logrado mediante metodologías activas. Este proyecto plantea como investigable la aplicación del aprendizaje colaborativo en Derecho Probatorio. Postula la planificación de la asignatura adecuándose a determinados parámetros descriptivos, taxonómicos, de resultados y métodos para cumplirlos y evaluarlos. Es posible implementar la recomendación de Julio Cortázar con el maestro que insta a asumir la tarea de instruir y educar, dando alas y orientando a esa conciencia naciente del alumno. 


\section{Referencias}

Alford, R. (2008). How Do You Trim the Seamless Web? Considering the Unintended Consequences of Pedagogical Alterations. University of Cincinnati Law Review, 77, 1273.

Anderson, L. W., Krathwohl, D. R., Airasian, P. W., Cruikshank, K. A., Mayer, R. E., Pintrich, P. R., Raths, J. y Wittrock, M. C. (2001). A taxonomy for learning, teaching, and assessing: A revision of Bloom 's Taxonomy of Educational Objectives. New York: Longman.

Ayers, P. (2006). Using subjective measures to detect variations of intrinsic cognitive load within problems. Learning and Instruction, 16(5), 389-400.

Bartimote-Aufflick, K., A., B. y Ainle, M. (2006). University Teachers Engaged in Critical Self-Regulation: How May They Influence Their Students? En A. Efklides y P. Misailidi, Trends and Prospects in Metacognition Research (pp. 427-444). London: Springer.

Beryl, B. (1992). Teaching Evidence: Storytelling in the Classroom. The American University Law Review, 41, 453.

Berrett. D. (2012). How 'flipping' the classroom can improve the traditional lecture. The Chronicle of Higher Education, 12, 1-14

Carnelutti, F. (1982). La Prueba Civil. Buenos Aires: EJEA.

Cobas Cobiella, M. E. (2015). El aprendizaje cooperativo: una competencia imprescindible para la formación de juristas. Revista Boliviana de Derecho, 18, 604-621.

Cobas Cobiella, M. M. y Mirrow, M. (2014). Educación legal en los Estados Unidos. Facultades de Derecho y el Juris Doctor. Inter-American Law Review , 46(1), 7-31.

Coloma Correa, R. (2005). El ocaso del profesor Binns: Un ensayo acerca de la enseñanza del derecho en Chile. Ius et Praxis, 11(1), 133-172. 
Couture, E. (1977). Fundamentos del Derecho Procesal Civil. Buenos Aires: Depalma.

Fiss, O. (1999). El derecho según Yale. En M. Böhmer, La enseñanza del Derecho y el ejercicio de la abogacía (págs. 26-35). Barcelona: Gedisa.

Fadul, J. A. (2009). Collective Learning: Applying distributed cognition for collective intelligence. The International Journal of Learning. 16 (4), 211-220.

Fernández March, A. y Bolonia, T. (2006). Metodologías activas para la formación de competencias. México, D.F.: Educación Siglo XXI.

Fredrick E. (2010). Toward a General Theory of Standards of Proof, 60 Cath. U. L. Rev. (2010), 1.

Gokhale, A. A. (1995). Collaborative learning enhances critical thinking. Journal of Technology Education, 7(1), 22-30.

Hammill, J., Best, G. y Anderson, J. (2015). Developing Student Mentor selfregulation skills through formative feedback: Rubric development phase. Journal of Peer Learning, 8, 48-58.

Heer, R. (2012). Iowa State University Center for Excellence in Learning and Teaching Updated. Recuperado de http://www.celt.iastate.edu/teaching/effective-teaching-practices/revised-blooms-taxonomy(revisado 25/8/2016).

Hess, Gerald F. (2013). Blended Courses in Law School: The Best of Online and Face-to- Face Learning? McGeorge Law Review, 45, 51-67

Hess, G., Friedland, S., Schwartz, M. y Sparrow, S. (2011). Techniques for Teaching Law 2. North Carolina: Carolina Academic Press.

Hopkins, A. (2008). Teaching Evidence Law Within the Framework of a Trial: Relating Theory to Practice as Students Take to Their Feet and Take Responsibility for the Trial Narrative. Journal of the Australian Law Teachers Association, 173-184. 
Iborra Cuéllar, A. y Izquierdo Alonso, M. (2010). ¿Cómo afrontar la evaluación del aprendizaje colaborativo? Una propuesta valorando el proceso, el contenido y el producto de la actividad grupal.Revista General de Información y Documentación, 20, 221-241.

Kaori, Y. y Hidetoshi, Y. (2017). Project-based learning in out-of-class activities: flipped learning based on communities created in real and virtual spaces. Procedia Coputer Service, 112, 1044-1053.

Kennedy, D. (1987). The Responsibility of Lawyers for the Justice of Their Causes. Texas Tech Law Review, 18, 1157-1163.

Kennedy, D. (1982). Legal Education and the Reproduction of Hierarchy. Journal of Legal Education. 591-615.

Lobato, C. (1997). Hacia una comprensión del aprendizaje cooperativo. Revista de Psicodidáctica, 4, 59-76.

Kolb, D. A. (1976). The Learning Style Inventory: Technical Manual, Boston, Ma.: McBer.

Kolb, A. Y., y Kolb, D. A. (2005). The Kolb Learning Style Inventory - Version 3.1: 2005 Technical Specifications. Haygroup: Experience New York: Based Learning Systems Inc.

Lage, M. J., Platt, G. J. y Treglia, M. (2000). Inverting the classroom: A gateway to creating aninclusive learning environment. The Journal of Economic Education, 31(1), 30-43.

Lam, D. (2004). Problem-based learning: an integration of theory and field. Journal of Social Work Education, 40, 371, 372.

Leclerc, O. (2012). La distinction entre 'la preuve en droit' et la 'preuve en science' est-elle pertinente? E. Truilhé-Marengo. Preuve scienti que, preuve juridique. Paris: Larcier. 
Lemmer, C. (2013). A view from the Flip Side: Using the "Inverted Classroom" to Enhance the Legal Information Literacy of the International LL.M. Student. Law Library Review, 2, 461-491.

Mayer, R. E. (2009). Learning and Instruction. Upper Saddle River, NJ.: Pearson/ Merrill/ Prentice Hall.

Mazur, E. (1996). Peer Instruction: A User's Manual. Boston, MA: Addison Wesley.

Mazur, E. (2006). Peer Instruction: Wie man es schafft, Studenten zum Nachdenken zu bringen. Praxis der Naturwissenschaften. Physik in der Schule, 55(4), 11-15.

Miller, K., Schell, J., Ho, A., Lukoff, B. y Mazur, E. (2015). Response switching and self efficacy inPeer Instruction classrooms. Physical Review Special Topics - Physics Education Research, 11(1),1-8.

Nicol, D. J. y Boyle, J. T. (2003). Peer instruction versus class- wide discussion in large classes: A comparison of two interaction methods in the wired classroom. Studies Higher Education, 28, 457-473.

Nance, D. (2016). The Burden of Proof. Cambridge: Cambridge U. Press.

Oh, R. y Reamy, B. (2014). The Socratic Method and Pimping: Optimizing the Use of Stress and Fear in Instruction. American Medical Association Journal of Ethics, 16(3), 182-186.

Orejundo-Hernández, S., Fernández-Turrado, T. y Garrido-Laporte, M. A. (2008). Experiencias con metodologías activas en la formación del profesorado. Revista Interuniversitaria de Formación del Profesorado, 63(22), 21-45.

Palincsar, A. S. (1998). Social constructivist perspectives on teaching and learning. Annual Reviewof Psychology, 49, 345-373.

Prince, M. (2004). Does active learning work? A review of the research. Journal of Engineering Education, 93(3), 223-231. 
Pantoja Ospina, M. A. (2013). Learning Styles Models : An upgrade for their revision and analysis. Revista Colombiana de Educación, (64), 79-105.

Posner, R. A. (2001). Clinical and Theoretical Approaches to the Teaching of Evidence and Trial Advocacy. Chicago: University of Chicago Law School

Prütting, H. (2016). Münchener Kommentar zur ZPO. Múnich: Beck.

Ramy, H. (2013). Moving Students from Hearing and Forgetting to Doing and Understanding: A Manual for Assessment in Law School. Capital University Law Review, 41, 837-850.

Roberts, P. (2002). Rethinking the Law of Evidence: A Twenty-First Century Agenda for Teaching and Research. Current Legal Problems, 55(1), 1.

Rothstein, P. (2006). Teaching Evidence. Georgetown: Georgetown University Law Center.

Ryan, E., Shuai, X., Ye, Y. y Ran, Y. (2014). When Socrates meets Confucius: teaching creative and critical thinking across cultures through multilevel Socratic method. Nebraska Law Review, 92(2), 290-349.

Santiveri Morata, F., Iglesias Rodríguez, C., Gil Iranzo, R. y Rourera Jordana, R. (2011). Metodologías activas en la docencia universitaria: resultados de algunas experiencias realizadas. IX Jornades de xarxes d'investigació en docència universitària Recuperado de http://dialnet.unirioja.es/servlet/articulo? codigo $=4127817$

Sentis Melendo, S. (1979). La Prueba. Buenos Aires: EJEA.

Schatzberg, L. (1999). Applying Bloom's and Kolb's Theories To Teaching Systems Analysys and Design. Recuperado de https:/www.researchgate.net/publication/241724614_Applying_Bloom's_and_Kolb's_Theories_To_Teaching_Systems_Analysis_Design 
Strayer, J. F. (2012). How learning in an inverted classroom influences cooperation, innovation and task orientation. Learning Environments Research, 15(2), 171-180.

Taruffo, M. (2003). Rethinking the Standard of Proof. American Journal of Comparative Law, 51, 659-677.

Taruffo, M. (2010). Evidence, in International Encyclopedia of Comparative Law, Vol. XVI, Cap. 7, Berlin: Mohr Siebeck, Tübingen.

Thalluri, J., O 'Flaherty, J. A. y Shepherd, P. L. (2014). Classmate peer-coaching: "A Study BuddySupport scheme". Journal of Peer Learning, 7(7), 92-104.

Turull, M. (Ed). (2011). Experiencias de mejora e innovación docente en el ámbito del Derecho. Barcelona: Universidad de Barcelona.

Thies, D. (2010). Rethinking Legal Education in Hard Times: The Recession, Practical Legal Education and the New Job Market. Journal of Legal Education, 59, 598-630.

Vickrey, T., Rosploch, K., Rahmanian, R., Pilarz, M. y Stains, M. (2015). Research- Based Implementation of Peer Instruction: A Literature Review. Life Sciences Education, 2, 14-19.

Wolff, L.-C. y Chan, J. (2016). Flipped Classrooms for Legal Education. New York: Springer.

\section{Notas}

${ }^{1}$ No se profundiza en la definición, concepto o descripción de lo que por "pedagogía" o "didáctica". No es el objeto de este texto, sino aplicar terminología, conceptos y metodología de las Ciencias de la Educación que dicen relación con la pedagogía y didáctica. El texto puede catalogarse como perteneciente a la preocupación en los últimos veinte años sobre la renovación en la enseñanza del Derecho. 
${ }^{2}$ Importante para el lector no versado en Derecho como para el jurista es relevante esta parte. Para el primero debe describirse en núcleo esencial de la asignatura no reducido a enunciación de contenidos, sino esenciales. Para el segundo, sirve para explicar la visión del autor sobre el tema que es objeto de enseñanza.

3 Acá se describe no ya la esencia de la prueba (tratada en el acápite anterior), sino que se describe el programa de la asignatura en sus contenidos y tiempos para esta parte introductoria en la que se aplicaron estas propuestas metodológicas innovativas.

${ }^{4}$ Por desarrollo se entiende la enunciación y examen de cada paso, detalle, secuencia que intenta aplicar la metodología colaborativa con el diálogo socrático en base a problemas y casos que emanan de la narrativa del film empleado.

\section{Anexo 1}

\section{Propuesta para la innovación enseñanza aprendizaje: marcos teóricos}

Desarrollo de la parte inicial de la asignatura (programa formulado según Bloom en resultados y habilidades (Macro)) la propuesta de este proyecto solo se aplica a las primeras unidades.

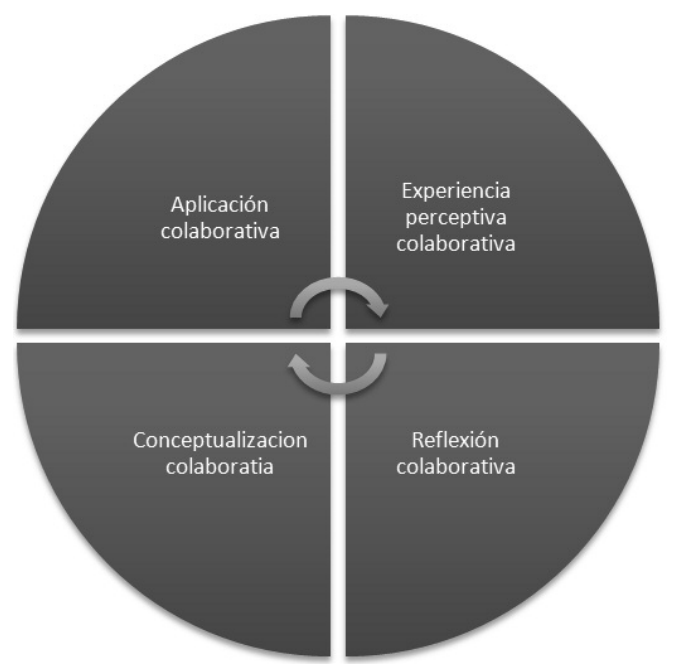

Revista de la Facultad de Derecho, (45), 2018, e20184506 DOI:10.22187/rfd2018n45a6 ISSN 0797-8316 / eISSN 2301-0665 

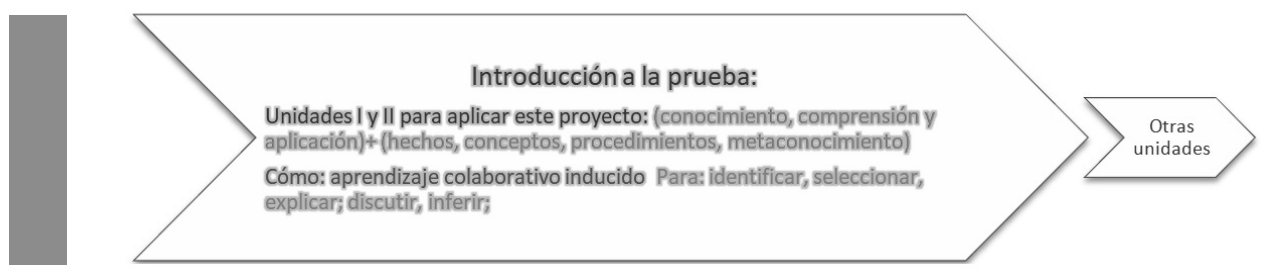

\section{Anexo 2}

Secuencia de actividades de enseñanza y evaluativas, resultados esperados, relación con el contenido del programa y actividad a realizar

\section{Trabajos en grupo Primera sesión película (1 $1^{\mathrm{a}}$. Parte)}

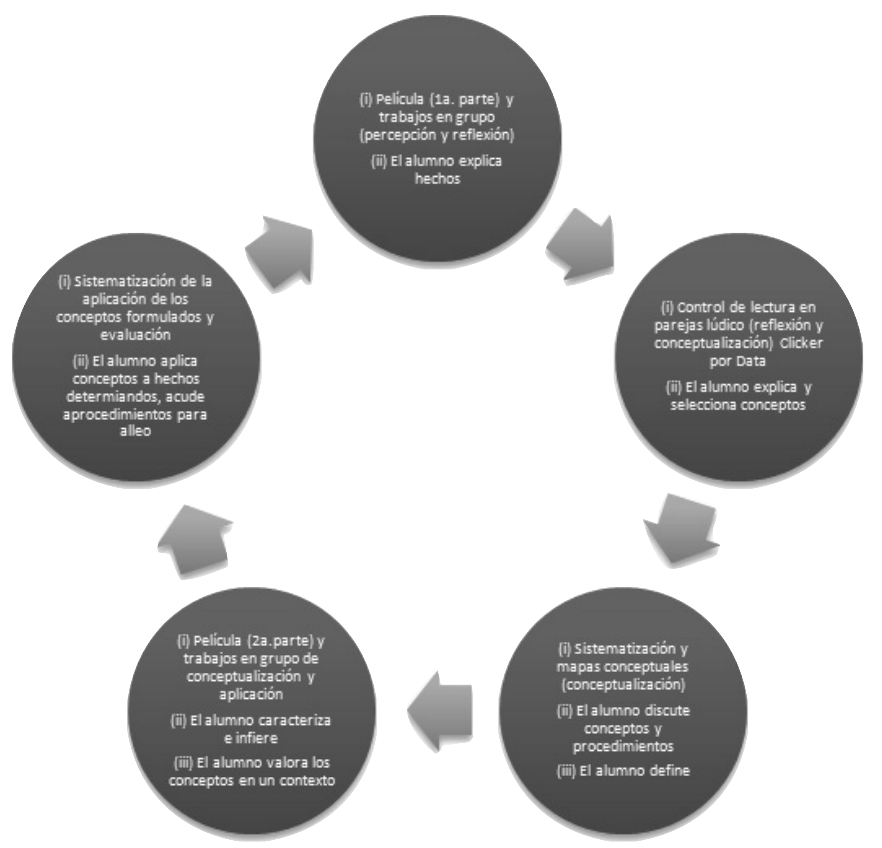

Instrucciones

Ejemplo Grupo 1

Discuta en grupo sobre la película y complete lo solicitado en la matriz. Luego un miembro del grupo expondrá el resultado.

1. Describa lo acontecido de acuerdo a la visión del demandante ¿Cómo sucedieron los hechos según él?

2. Describa lo acontecido de acuerdo a la visión del demandado ¿Cómo sucedieron los hechos según él? 
3. Señale las coincidencias entre las dos visiones. ¿Sobre qué detalles coinciden las dos visiones?

\begin{tabular}{|l|l|l|l|}
\hline & Qué y cómo & Cuándo & Dónde \\
\hline $\begin{array}{l}\text { Acontecimientos según } \\
\text { demandante }\end{array}$ & & & \\
\hline $\begin{array}{l}\text { Acontecimientos según } \\
\text { demandando }\end{array}$ & & & \\
\hline Señale las coincidencias & & & \\
\hline
\end{tabular}

\section{Trabajos en grupo cuarta sesión película (2ª Parte)}

\section{Ejemplo Grupo 1}

Discuta en grupo sobre el texto entregado para lectura y tome nota de las respuestas que propongan los otros grupos que se relacionen con la matriz que deben completar. Complete la matriz y luego un miembro del grupo las expondrá.

\begin{tabular}{|c|c|c|}
\hline Objeto de la prueba & $\begin{array}{c}\text { Definición de } \\
\text { prueba judicial }\end{array}$ & Medios de prueba \\
\hline ¿Qué se prueba? & ¿Qué es la prueba? & ¿Con qué se prueba? \\
\hline $\begin{array}{c}\text { ¿Qué no necesita } \\
\text { probarse? }\end{array}$ & $\begin{array}{c}\text { ¿Cuál es la finalidad } \\
\text { de la prueba? }\end{array}$ & $\begin{array}{c}\text { ¿Cuáles son las } \\
\text { medios de prueba? }\end{array}$ \\
\hline
\end{tabular}

\section{Anexo 3}

\section{Quiz, actividad evaluativa con nota}
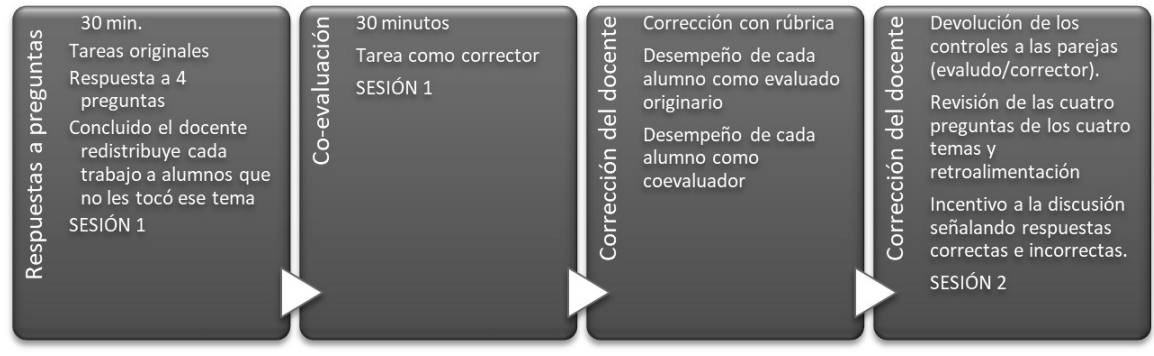

Revista de la Facultad de Derecho, (45), 2018, e20184506

DOI:10.22187/rfd2018n45a6

ISSN 0797-8316 / elSSN 2301-0665

29 de 29 